\title{
Dominica Islander
}

National Cancer Institute

\section{Source}

National Cancer Institute. Dominica Islander. NCI Thesaurus. Code C43824.

Denotes the inhabitants of Dominica, a person from there, or their descendants elsewhere. 